\title{
A CHARACTERIZATION OF POSITIVE QUADRATURE FORMULAE
}

\author{
YUAN XU
}

\begin{abstract}
A positive quadrature formula with $n$ nodes which is exact for polynomials of degree $2 n-r-1,0 \leq r \leq n$, is based on the zeros of certain quasi-orthogonal polynomials of degree $n$. We show that the quasi-orthogonal polynomials that lead to the positive quadrature formulae can all be expressed as characteristic polynomials of a symmetric tridiagonal matrix with positive subdiagonal entries. As a consequence, for a fixed $n$, every positive quadrature formula is a Gaussian quadrature formula for some nonnegative measure.
\end{abstract}

\section{INTRODUCTION}

Let $\alpha$ be a nonnegative measure on the real line $\mathbb{R}$ with an infinite support and finite moments. For $n \in \mathbb{N}$ and $0 \leq r \leq n$, a linear functional

$$
\mathscr{I}_{n, r}(d \alpha ; f)=\sum_{k=1}^{n} \lambda_{k, n} f\left(x_{k, n}\right), \quad \lambda_{k, n} \in \mathbb{R}, x_{k, n} \in \mathbb{R}
$$

is called a $(2 n-1-r, n)$ quadrature formula for $d \alpha$ if

$$
\int_{-\infty}^{\infty} p(x) d \alpha=\mathscr{I}_{n, r}(d \alpha ; p), \quad p \in \Pi_{2 n-r-1},
$$

where $\Pi_{n}$ is the set of polynomials of degree at most $n$. The numbers $\left\{x_{k, n}\right\}$ are called nodes, and the $\left\{\lambda_{k, n}\right\}$ weights, of the quadrature formula. When all quadrature weights are positive, $\mathscr{I}_{n, r}(d \alpha ; f)$ is a positive linear functional; we call it a positive $(2 n-1-r, n)$ quadrature formula. The Gaussian quadrature is the unique $(2 n-1, n)$ formula, and it is positive. It is well known that $(2 n-1-r, n)$ formulae are related to quasi-orthogonal polynomials. Let $p_{n}(d \alpha)$ denote the orthonormal polynomial of precise degree $n$ with respect to $d \alpha$. Let $\rho_{1}, \ldots, \rho_{r}$ be real numbers. Then the quasi-orthogonal polynomials of degree $n$, order $r$, are defined by

$$
q_{n, r}(d \alpha)=p_{n}(d \alpha)+\rho_{1} p_{n-1}(d \alpha)+\cdots+\rho_{r} p_{n-r}(d \alpha), \quad \rho_{r} \neq 0 .
$$

For convenience we write $q_{n, 0}=p_{n}$. It is easy to see that the nodes of a $(2 n-1-r, n)$ quadrature formula (1.1) are the distinct zeros of a quasiorthogonal polynomial $q_{n, r}(d \alpha)$. On the other hand, if a quasi-orthogonal polynomial $q_{n, r}(d \alpha)$ has $n$ distinct real zeros, then there is a $(2 n-1-r, n)$ quadrature formula based on these zeros provided the quadrature weights come

Received by the editor March 18, 1993.

1991 Mathematics Subject Classification. Primary 65D32, 33C45, 41 A05.

Key words and phrases. Quadrature formula, quasi-orthogonal polynomial, tridiagonal matrix. 
out to be nonzero. We call such a quadrature formula generated by the quasiorthogonal polynomial $q_{n, r}$.

The $(2 n-1-r, n)$ quadature formulae have been studied by several authors (cf. [3, 6, 7, 8, 9, 12] and the references therein). In particular, if $\alpha$ has as support the interval $[-1,1]$, then all $(2 n-r-1, n)$ positive quadrature formulae with nodes in $[-1,1]$ were characterized in [6] for $r=2$, and in [7, 8, 9] for all $r$. The case $r=1$ is known already in [10, p. 46]. In [12], we studied the case $r=2,3$ by means of a new representation of quasiorthogonal polynomials. We found there that a large class of quasi-orthogonal polynomials can be represented as the characteristic polynomials of symmetric tridiagonal matrices. As a consequence, the zeros of quasi-orthogonal polynomials are recognized as eigenvalues of these matrices, and thus can be studied by making use of the techniques developed for eigenvalue problems. Based on this method, we proved a number of results in [12] concerning quadrature formulae and interpolating polynomials. However, not all quasi-orthogonal polynomials have such a representation. For those that do, the entries of the symmetric tridiagonal matrix used in the representation depend on the coefficients $\rho_{k}$ in (1.1) nonlinearly, and their formulae become very complicated for large $r$. On the other hand, from a numerical point of view, it is very desirable to have the quasi-orthogonal polynomial given as a characteristic polynomial of a symmetric tridiagonal matrix, which allows the use of efficient numerical methods for studying the zeros of such a polynomial, and for the quadrature formula generated by the polynomial.

The purpose of this paper is to continue the study initiated in [12], and we shall base our study on the quasi-orthogonal polynomials expressible through symmetric tridiagonal matrices. One of our main results states that every positive $(2 n-r-1, n)$ quadrature formula is generated by a quasi-orthogonal polynomial that has a symmetric tridiagonal matrix representation with positive subdiagonal entries. As a consequence, the nodes of every positive quadrature can be interpreted as eigenvalues of a symmetric matrix, thus, can be easily computed as such. Moreover, we will also derive explicit and simple formulae for quadrature weights. Hence, an efficient numerical method of constructing positive quadrature formulae is almost immediate. Another interesting result that we shall prove states that every positive quadrature formula, for a fixed $n$, can be realized as a Gaussian quadrature for another nonnegative measure, which usually depends on $n$.

The paper is organized as follows. In the next section we fix notation and provide the preliminaries. In $\S 3$, we discuss the representation of quasi-orthogonal polynomials by means of tridiagonal matrices. The characterization of positive quadrature formulae is presented in $\S 4$. Finally, in $\S 5$, we discuss the construction of positive quadrature formulae, and the location of the quadrature nodes.

\section{Preliminaries}

Let $\Pi$ be the set of all polynomials, and $\Pi_{n}$ the set of polynomials of degree at most $n$. Let $\mathscr{L}$ be a linear functional defined on $\Pi$. We call $\mathscr{L}$ positive if $\mathscr{L}(p) \geq 0$ for every nonnegative $p \in \Pi$, and $\mathscr{L}(p)=0$ only if $p=0$. From the moment theory it is known (cf. [1]) that every positive linear functional admits an integral representation with respect to a nonnegative measure $\alpha$, 
such that $\alpha^{\prime}>0$ a.e. and

$$
\mathscr{L}(f)=\int f(x) d \alpha, \quad f \in \Pi .
$$

Let $\mathscr{M}$ be the class of measures $\alpha$ on $\mathbb{R}$ with an infinite support and finite moments such that $\alpha^{\prime}>0$ almost everywhere in its support set. For $\alpha \in \mathscr{M}$, normalized so that $\int d \alpha=1$ for convenience, we denote by $\left\{p_{n}(d \alpha)\right\}_{n=0}^{\infty}$ a sequence of orthonormal polynomials with respect to $d \alpha$,

$$
\int_{-\infty}^{\infty} p_{n}(d \alpha, x) p_{m}(d \alpha, x) d \alpha=\delta_{n, m}, \quad p_{n}(d \alpha, x)=\gamma_{n}(d \alpha) x^{n}+\cdots
$$

where $\gamma_{n}(d \alpha)>0$ for all $n \in \mathbb{N}_{0}$. For our study of the quasi-orthogonal polynomials we shall need a number of properties of orthogonal polynomials. All of them can be found in standard books on orthogonal polynomials, such as $[1,3,10]$. We begin with

The three-term relation. There exist sequences of real numbers $a_{n}>0$ and $b_{n}$ such that

$$
x p_{n}(d \alpha)=a_{n} p_{n+1}(d \alpha)+b_{n} p_{n}(d \alpha)+a_{n-1} p_{n-1}(d \alpha), \quad n \geq 1,
$$

where $p_{-1}(d \alpha)=0$ and $p_{0}(d \alpha)=1$.

In this equation we wrote $a_{n}$ for $a_{n}(d \alpha)$. As a rule, whenever there is no danger of confusion, we shall omit $d \alpha$ from our notation. For example, we shall write $\gamma_{n}$ for $\gamma_{n}(d \alpha)$. The coefficients $a_{n}$ in the three-term relation and the leading coefficients $\gamma_{n}$ of $p_{n}(d \alpha)$ are related by

$$
a_{n}=\frac{\gamma_{n}}{\gamma_{n+1}}, \quad \text { or } \quad \gamma_{n}^{-1}=a_{0} \cdots a_{n-1} \text {. }
$$

Moreover, the three-term relation actually characterizes the orthogonality, which is the content of

Favard's Theorem. Let $\left\{p_{n}\right\}_{n=0}^{\infty}, p_{0}=1$, be a sequence of polynomials, $p_{n} \in \Pi_{n}$. Then $\left\{p_{n}\right\}_{n=0}^{\infty}$ is orthonormal with respect to $d \mu$ for some $\mu \in \mathscr{M}$ if and only if it satisfies a three-term relation with $a_{n}>0$ and $b_{n} \in \mathbb{R}, n \in \mathbb{N}_{0}$.

The coefficients in the three-term relation defines a tridiagonal matrix, called the Jacobi matrix, with $b_{n}$ on the main diagonal and $a_{n}$ on the subdiagonals. This matrix plays an important role in the theory of orthogonal polynomials. We have, for example,

Representation of orthonormal polynomials. Let $J_{n}$ be the truncated Jacobi matrix

$$
J_{n}=\left[\begin{array}{ccccc}
b_{0} & a_{0} & & & 0 \\
a_{0} & b_{1} & a_{1} & & \\
& \ddots & \ddots & \ddots & \\
& & & b_{n-2} & a_{n-2} \\
0 & & & a_{n-2} & b_{n-1}
\end{array}\right]
$$

Then

$$
p_{n}(d \alpha)=\gamma_{n} \operatorname{det}\left(x I-J_{n}\right),
$$

where $\gamma_{n}$ is the leading coefficient of $p_{n}$ and $I$ is the identity matrix. 
From this representation, all zeros of $p_{n}(d \alpha)$ are eigenvalues of the matrix $J_{n}$. We denote these zeros by $x_{k, n}=x_{k, n}(d \alpha)$, and assume the following order: $x_{1, n}<x_{2, n}<\cdots<x_{n, n}$. The properties of the zeros of $p_{n}(d \alpha)$ are collected in the following statement.

Properties of zeros. All zeros of $p_{n}$ are real and distinct. If $\alpha$ has compact support $[a, b]$, then all zeros are located inside $[a, b]$. The zeros of $p_{n}(d \alpha)$ and $p_{n+1}(d \alpha)$ interlace: each interval $\left[x_{k, n}, x_{k+1, n}\right]$ contains exactly one zero of $p_{n+1}$, where $x_{0, n}=a$ and $x_{n+1, n}=b$.

If $\operatorname{supp} \alpha=[a, b]$, then because $\gamma_{n}>0$ and $p_{n}(d \alpha)=\gamma_{n} x^{n}+\cdots$, we have

$$
\operatorname{sign} p_{k}(a)=(-1)^{k} \quad \text { and } \operatorname{sign} p_{k}(b)=1 .
$$

Another property of $p_{n}(d \alpha)$ that follows from the three-term relation is the Christoffel-Darboux formula. Let $K_{n}(d \alpha, \cdot, \cdot)$ be defined by

$$
K_{n}(d \alpha, x, y)=\sum_{k=0}^{n-1} p_{k}(d \alpha, x) p_{k}(d \alpha, y),
$$

which is the reproducing kernel function of $\left\{p_{n}(d \alpha)\right\}$. Then we have

Christoffel-Darboux formula. For $n \in \mathbb{N}$, there holds

$$
K_{n}(d \alpha, x, y)=a_{n-1} \frac{p_{n}(x) p_{n-1}(y)-p_{n}(y) p_{n-1}(x)}{x-y} .
$$

For the Gaussian quadrature formula with respect to $d \alpha$, the quadrature weights are given in terms of $K_{n}(d \alpha)$ as

$$
\lambda_{k, n}=\left[K_{n}\left(d \alpha, x_{k, n}, x_{k, n}\right)\right]^{-1},
$$

where $\lambda_{k, n}$ is the weight corresponding to the node $x_{k, n}$. This ends our preliminaries.

There are other definitions to be given later. The most important one is the symmetric matrix representation of quasi-orthogonal polynomials, which will be given after the proof of Theorem 3.1.

\section{THE QUASI-ORTHOGONAL POLYNOMIALS}

For the quasi-orthogonal polynomial $q_{n, r}$ defined as in (1.3), it is clear that $q_{n, r}$ is orthogonal to the space $\Pi_{n-r-1}$. This fact is sometimes taken to be the definition of the quasi-orthogonal polynomials of order $r$, since every polynomial orthogonal to $\Pi_{n-r-1}$ can be expressed in the form of (1.3). From this point of view, the formula (1.3) seems to be the natural starting point for the study of the quasi-orthogonal polynomials, and it is indeed so in the literature. In this section, we shall present a different way of dealing with quasi-orthogonal polynomials, which shows that many of these polynomials can be represented as characteristic polynomials of symmetric tridiagonal matrices. This representation has been considered only recently in [12]. We begin with 
Theorem 3.1. Let $\alpha \in \mathscr{M}$ and let $T_{n, 2 s-1}$ be a matrix defined by

$$
T_{n, 2 s-1}=\left[\begin{array}{ccccc}
b_{0}^{*} & a_{0}^{*} & & & 0 \\
a_{0}^{*} & b_{1}^{*} & a_{1}^{*} & & \\
& \ddots & \ddots & \ddots & \\
& & & b_{n-2}^{*} & a_{n-2}^{*} \\
0 & & & a_{n-2}^{*} & b_{n-1}^{*}
\end{array}\right]
$$

where $a_{k}^{*}=a_{k}(d \alpha), 0 \leq k \leq n-s-1, b_{k}^{*}=b_{k}(d \alpha), 0 \leq k \leq n-s-1$, and

$$
\begin{aligned}
& a_{k}^{*}=a_{k}(d \alpha)\left(1-\tau_{k+s-n+1}\right), \quad n-s \leq k \leq n-2, \\
& b_{k}^{*}=b_{k}(d \alpha)-a_{k}(d \alpha) \sigma_{k+s-n+1}, \quad n-s \leq k \leq n-1,
\end{aligned}
$$

and $\sigma_{k}, 1 \leq k \leq s$, and $\tau_{k}, 1 \leq k \leq s-1$, are real numbers. Also let $T_{n, 2 s-2}$ be the matrix $T_{n, 2 s-1}$ with $\sigma_{1}=0$, and take $T_{n, 0}=J_{n}$. Then the characteristic polynomial of $T_{n, r}, r=2 s-1$ or $2 s-2$, is a quasi-orthogonal polynomial,

$$
\begin{aligned}
q_{n, r}(d \alpha, x) & =\gamma_{n} \operatorname{det}\left(x I-T_{n, r}\right) \\
& =p_{n}(d \alpha)+\rho_{1} p_{n-1}(d \alpha)+\cdots+\rho_{r} p_{n-r}(d \alpha),
\end{aligned}
$$

where $\rho_{1}, \ldots, \rho_{r}$ are functions, in general nonlinear, of $\tau_{1}, \ldots, \tau_{s-1}$ and $\sigma_{1}, \ldots, \sigma_{s}$.

Proof. Since $T_{n, 2 s-1}$ becomes $T_{n, 2 s-2}$ when $\sigma_{1}=0$, we use induction on $T_{n, 2 s-1}$. For $s=1$ we have

$$
\begin{aligned}
q_{n, 1}(d \alpha, x) & =\gamma_{n} \operatorname{det}\left(x I-J_{n}+\left[\begin{array}{ll}
0 & \\
& a_{n-1} \sigma_{1}
\end{array}\right]\right) \\
& =p_{n}(d \alpha, x)+\gamma_{n} a_{n-1} \sigma_{1} \operatorname{det}\left(x I-J_{n-1}\right) \\
& =p_{n}(d \alpha, x)+\sigma_{1} p_{n-1}(d \alpha, x),
\end{aligned}
$$

where if $\sigma_{1}=0$, then we have $q_{n, 0}(d \alpha, x)=p_{n}(d \alpha, x)$. In general, we write $q_{n, r}(d \alpha, x)=\gamma_{n} \operatorname{det}\left(x I-T_{n, r}\right)=p_{n}(d \alpha)+\rho_{1, r}^{(n)} p_{n-1}(d \alpha)+\cdots+\rho_{r, r}^{(n)} p_{n-r}(d \alpha)$, where $\rho_{j, r}^{(n)}=\rho_{j}^{(n)}\left(\sigma_{1}, \ldots, \sigma_{r}, \tau_{1}, \ldots, \tau_{r-1}\right), 1 \leq j \leq r$. Suppose (3.2) has been proved for $T_{n, 2 i-1}$ for $i \leq s$. We now prove (3.2) for $r=2 s+1$. By the definition of $T_{n, r}$ we have

$$
T_{n, 2 s+1}=J_{n}-\left[\begin{array}{cccccc}
0 & & & & & 0 \\
& 0 & a_{n-s} \sigma_{1} & a_{n-s} \tau_{1} & & \\
& & a_{n-s} \tau_{1} & a_{n-s+1} \sigma_{2} & a_{n-s+1} \tau_{2} & \\
& \ddots & \ddots & \ddots & \\
& & & a_{n-3} \tau_{s-2} & a_{n-2} \sigma_{s-1} & a_{n-2} \tau_{s-1} \\
0 & & & & a_{n-2} \tau_{s-1} & a_{n-1} \sigma_{s}
\end{array}\right] .
$$


Expanding the determinant $\operatorname{det}\left(x I-T_{n, 2 s+1}\right)$ by its last row, we have

$$
\begin{aligned}
q_{n, 2 s+1}(d \alpha)= & \gamma_{n}\left(x-b_{n-1}+a_{n-1} \sigma_{s+1}\right) \operatorname{det}\left(x I-T_{n-1,2 s-1}\right) \\
& +\gamma_{n} a_{n-2}^{2}\left(1-\tau_{s}\right)^{2} \operatorname{det}\left(x I-T_{n-2,2 s-3}\right) \\
= & a_{n-1}^{-1}\left(x-b_{n}+a_{n-1} \sigma_{s+1}\right) q_{n-1,2 s-1}(d \alpha) \\
& +a_{n-1}^{-1} a_{n-2}\left(1-\tau_{s}\right)^{2} q_{n-2,2 s-3}(d \alpha) \\
= & a_{n-1}^{-1}\left(x-b_{n}+a_{n-1} \sigma_{s+1}\right) \\
& \times\left[p_{n-1}(d \alpha)+\rho_{1,2 s-1}^{(n-1)} p_{n-2}(d \alpha)+\cdots+\rho_{2 s-1,2 s-1}^{(n-1)} p_{n-2 s}(d \alpha)\right] \\
& +a_{n-1}^{-1} a_{n-2}\left(1-\tau_{s}\right)^{2} \\
& \times\left[p_{n-2}(d \alpha)+\rho_{1,2 s-3}^{(n-2)} p_{n-3}(d \alpha)+\cdots+\rho_{2 s-3,2 s-3}^{(n-2)} p_{n-2 s+1}(d \alpha)\right],
\end{aligned}
$$

where we have used the induction hypothesis. Let $\rho_{0, t}^{(m)}=1$. We can rewrite the above formula and use the three-term relation to get

$$
\begin{aligned}
q_{n, 2 s+1}(d \alpha)= & a_{n-1}^{-1} \sum_{k=0}^{2 s-1}\left(x-b_{n-1-k}\right) \rho_{k, 2 s-1}^{(n-1)} p_{n-k-1}(d \alpha) \\
& +a_{n-1}^{-1} \sum_{k=0}^{2 s-1}\left(b_{n-1-k}-b_{n-1}-a_{n-1} \sigma_{s+1}\right) \rho_{k, 2 s-1}^{(n-1)} p_{n-1-k}(d \alpha) \\
& +a_{n-1}^{-1} a_{n-2}\left(1-\tau_{s}\right)^{2} \sum_{k=0}^{2 s-3} \rho_{k, 2 s-3}^{(n-2)} p_{n-2-k}(d \alpha) \\
= & a_{n-1}^{-1} \sum_{k=0}^{2 s-1} \rho_{k, 2 s-1}^{(n-1)}\left(a_{n-k-1} p_{n-k}(d \alpha)\right. \\
& +a_{n-1}^{-1} \sum_{k=1}^{2 s} \rho_{k-1,2 s-1}^{(n-1)}\left(b_{n-k} \sum_{k=2}^{2 s+1} \rho_{k-2,2 s-1}^{(n-1)} a_{n-k} p_{n-k}(d \alpha)\right) \\
& \left.+a_{n-1}^{-1} a_{n-2}\left(1-\tau_{s}\right)^{2} \sum_{k=2}^{2 s-1} \rho_{k-2,2 s-3}^{(n-2)} \sigma_{s+1}\right) p_{n-k}(d \alpha)
\end{aligned}
$$

which is in the desired form (3.2) with

$$
\rho_{0,2 s+1}^{(n)}=\rho_{0,2 s-1}^{(n-1)}=1,
$$

and

$$
\begin{aligned}
\rho_{k, 2 s+1}^{(n)}=a_{n-1}^{-1}\left[\rho_{k, 2 s-1}^{(n-1)} a_{n-k-1}\right. & +\rho_{k-2,2 s-1}^{(n-1)} a_{n-k} \\
& +\rho_{k-1,2 s-1}^{(n-1)}\left(b_{n-k}-\right. \\
& \left.b_{n-1}-a_{n-1} \sigma_{s+1}\right) \\
& \left.+\rho_{k-2,2 s-3}^{(n-2)} a_{n-2}\left(1-\tau_{s}\right)^{2}\right]
\end{aligned}
$$

for $1 \leq k \leq 2 s$, and

$$
\rho_{2 s+1,2 s+1}^{(n)}=a_{n-1}^{-1} a_{n-2 s-1} \rho_{2 s-1,2 s-1}^{(n-1)},
$$


where we take $\rho_{j, k}^{(m)}=0$ if $j>k$ or $j<0$. In particular, we can iterate the last equation to conclude that

$$
\begin{aligned}
\rho_{2 s+1,2 s+1}^{(n)} & =a_{n-1}^{-1} \cdots a_{n-s}^{-1} a_{n-2 s-1} \cdots a_{n-s-1} \rho_{1,1}^{(n-s)} \\
& =a_{n-1}^{-1} \cdots a_{n-s}^{-1} a_{n-2 s-1} \cdots a_{n-s-1} \sigma_{1} .
\end{aligned}
$$

Therefore, if $\sigma_{1}=0$, then $\rho_{2 s+1,2 s+1}^{(n)}=0$ and $q_{n, 2 s+1}(d \alpha)$ becomes $q_{n, 2 s}(d \alpha)$. This concludes the proof.

A weaker version of this theorem appears in [12]. For $r=1$, every quasiorthogonal polynomial

$$
q_{n, 1}(d \alpha)=p_{n}(d \alpha)+\rho_{1} p_{n-1}(d \alpha)
$$

admits the representation (3.2) with $\sigma_{1}=\rho_{1}$. However, it is important to note that not every quasi-orthogonal polynomial has such a representation if $r>1$. For example, only those

$$
q_{n, 2}(d \alpha)=p_{n}(d \alpha)+\rho_{1} p_{n-1}(d \alpha)+\rho_{2} p_{n-2}(d \alpha)
$$

under the restriction $\rho_{2} \leq a_{n-2} / a_{n-1}$ admit a formula (3.2). For $r=2,3$ and those $q_{n, r}$ that admit a representation (3.2), formulae of $\sigma_{k}$ and $\tau_{k}$ in terms of $\rho_{k}$ are given in [12]. They are quoted in $\S 5$. The complexity of these formulae increases rapidly with $r$.

In the following we show that the class of polynomials given by (3.2) with $a_{k}^{*}>0$ is of special importance. For convenience, if a quasi-orthogonal polynomial $q_{n}$ has a determinant representation (3.2), then we say it has a symmetric matrix representation. If all subdiagonal elements $a_{k}^{*}>0$ in such a representation, we say that $q_{n}$ has a positive symmetric matrix representation.

We introduce the following notation. For $\alpha \in \mathscr{M}$ and $r<n$, we let $q_{n, r}$ be a quasi-orthogonal polynomials of degree $n$ and order $r$ and assume that $q_{n, r}$ has a symmetric matrix representation with matrix $T_{n, r}$. With respect to this fixed $q_{n, r}$ we define a sequence of quasi-orthogonal polynomials $q_{n-k, r-2 k}$ of degree $n-k$ and order $r-2 k$ by

$$
q_{n-k, r-2 k}=\gamma_{n-k} \operatorname{det}\left(x I-T_{n-k, r-2 k}\right), \quad 0 \leq k \leq\left[\frac{r}{2}\right],
$$

where $T_{n-k, r-2 k}$ is the $(n-k) \times(n-k)$ submatrix of $T_{n, r}$ at the left upper corner. We note that the definition of $q_{n-k, r-2 k}$ in (3.3) is consistent with (3.2). For convenience, we denote

$$
\begin{gathered}
Q_{k}=p_{k}(d \alpha), \quad 0 \leq k \leq n-\left[\frac{r}{2}\right]-1, \quad \text { and } \\
Q_{n-k}=q_{n-k, r-2 k}(d \alpha), \quad 0 \leq k \leq\left[\frac{r}{2}\right],
\end{gathered}
$$

and

$$
\begin{aligned}
P_{k} & =Q_{k}, \quad 0 \leq k \leq n-\left[\frac{r}{2}\right]-1, \quad \text { and } \\
P_{n-k} & =\left(1-\tau_{1}\right)^{-1} \cdots\left(1-\tau_{s-k}\right)^{-1} Q_{n-k}, \quad 0 \leq k \leq\left[\frac{r}{2}\right],
\end{aligned}
$$

where $r=2 s-1$ or $r=2 s-2$. We shall use these notations throughout the rest of the paper. By the similarity of (3.1) and (3.2) with the representation of orthogonal polynomials in (2.3) and (2.4), the following theorem seems natural. 
Theorem 3.2. Let $q_{n, r}$ be a quasi-orthogonal polynomial that has a positive symmetric matrix representation. Let $Q_{k}$ and $P_{k}$ be defined as above. Then $Q_{0}, \ldots, Q_{n}$ are the first $n+1$ members of a family of orthogonal polynomials. Moreover, $P_{0}, \ldots, P_{n}$ are the corresponding family of orthonormal polynomials. Proof. For $k<0$, let $\tau_{k}=\sigma_{k}=0$ and $T_{m, k}=J_{m}$. Let $r=2 s+1$. Then by the definition of $q_{n, k}$ we have

$$
\begin{aligned}
& \operatorname{det}\left(x I-T_{k+1,2(k+s-n+1)-1}\right) \\
& \quad=\left(x-b_{k}^{*}\right) \operatorname{det}\left(x I-T_{k, 2(k+s-n)-1}\right)-\left(a_{k-1}^{*}\right)^{2} \operatorname{det}\left(x I-T_{k-1,2(k+s-n-1)-1}\right),
\end{aligned}
$$

for $0 \leq k \leq n-1$. From this equality we obtain that the $Q_{k}$ satisfy the relation

$$
\gamma_{k+1}^{-1} Q_{k+1}=\left(x-b_{k}^{*}\right) \gamma_{k}^{-1} Q_{k}-\left(a_{k-1}^{*}\right)^{-1} \gamma_{k-1}^{-1} Q_{k-1}, \quad 0 \leq k \leq n-1 .
$$

Since $a_{k}^{*}=a_{k}\left(1-\tau_{k}\right)$, we obtain from this relation that the $P_{k}$ satisfy a threeterm relation

$$
x P_{k}=a_{k}^{*} P_{k+1}+b_{k}^{*} P_{k}+a_{k-1}^{*} P_{k-1}, \quad 0 \leq k \leq n-1,
$$

where $a_{n-1}^{*}=a_{n-1}$. Let $\left\{a_{k}^{*}\right\}, k \geq n$, be a sequence of positive numbers and $\left\{b_{k}^{*}\right\}, k \geq n$, be a sequence of real numbers. We can define $P_{k}, k \geq n$, by the recurrence formula

$$
P_{k}=\frac{1}{a_{k-1}^{*}}\left(x-b_{k-1}^{*}\right) P_{k-1}-\frac{a_{k-2}^{*}}{a_{k-1}^{*}} P_{k-2} .
$$

With this definition, the sequence of polynomials $\left\{P_{k}\right\}_{k=0}^{\infty}$ satisfies the threeterm relation

$$
x P_{k}=a_{k}^{*} P_{k+1}+b_{k}^{*} P_{k}+a_{k-1}^{*} P_{k-1}, \quad k \geq 0 .
$$

Since $a_{k}^{*}>0$ for all $k \geq 0$, by Favard's theorem, we conclude that $\left\{P_{n}\right\}_{n=0}^{\infty}$ is orthonormal with respect to a measure $\mu \in \mathscr{M}$.

We remark that it is very difficult to find explicitly the measure $\mu$ with respect to which $Q_{k}$ are orthogonal. Actually, this measure also depends on $n$ and the choices of $a_{k}^{*}$ and $b_{k}^{*}$ for $k>n$. In general, we should write $\mu=\mu_{n}$ to indicate the dependence of $\mu$ on $n$. Nevertheless, the following examples, in which $\mu$ is independent of $n$, seem to be of interest (cf. [1, p. 205]).

Let $T_{n}$ and $U_{n}$ be the Chebyshev polynomials of the first and the second kind, respectively. Let $b<1$. Then the quasi-orthogonal polynomials

$$
q_{1,1}(u, x)=x-b / 2, \quad q_{n, 1}(u, x)=U_{n}(x)-b U_{n-1}(x), \quad n \geq 2,
$$

with respect to the weight function $u(x)=\left(1-x^{2}\right)^{1 / 2}$ on $[-1,1]$ are orthogonal with respect to the weight function

$$
u^{*}(x)=\frac{\left(1-x^{2}\right)^{1 / 2}}{1+b^{2}-2 b x}, \quad-1<x<1 .
$$

And the quasi-orthogonal polynomials

$$
q_{n, 2}(x)=T_{n}(x)+\frac{a-1}{a+1} T_{n-2}(x), \quad a=\sqrt{1-b},
$$

with respect to the weight function $v(x)=\left(1-x^{2}\right)^{-1 / 2}$ on $[-1,1]$ are orthogonal with respect to the weight function

$$
v^{*}(x)=\frac{1}{\left(1-b x^{2}\right)\left(1-x^{2}\right)^{1 / 2}}, \quad-1<x<1 .
$$


Further discussions on the dependency of $\mu$ on $n$ is given after Theorem 4.4. There are several important corollaries of Theorem 3.2, which will have applications in the study of quadrature formulae.

Corollary 3.3. Let $q_{n, r}$ be a quasi-orthogonal polynomial that has a positive symmetric matrix representation. Then all zeros of $q_{n, r}$ are real and distinct. Moreover, the zeros of $q_{n, r}$ and $q_{n-1, r-2}$ mutually separate each other.

Corollary 3.4 (Christoffel-Darboux formula). Let $q_{n, r}$ be a quasi-orthogonal polynomial that has a positive symmetric matrix representation. Let

$$
\begin{aligned}
K_{n}^{*}(x, y)= & \sum_{k=0}^{n-1} P_{k}(x) P_{k}(y) \\
= & \sum_{k=0}^{n-[r / 2]-1} p_{k}(d \alpha, x) p_{k}(d \alpha, y) \\
& +\sum_{k=0}^{[r / 2]}\left(1-\tau_{1}\right)^{-2} \cdots\left(1-\tau_{s-k}\right)^{-2} q_{n-k, r-2 k}(x) q_{n-k, r-2 k}(y),
\end{aligned}
$$

where $r=2 s-1$ or $r=2 s-2$. Then for $x \neq y$,

$$
\begin{aligned}
K_{n}^{*}(x, y)= & a_{n-1}\left(1-\tau_{1}\right)^{-2} \cdots\left(1-\tau_{s-1}\right)^{-2} \\
& \cdot \frac{q_{n, r}(x) q_{n-1, r-2}(y)-q_{n, r}(y) q_{n-1, r-2}(x)}{x-y},
\end{aligned}
$$

and for $x=y$,

$$
\begin{aligned}
K_{n}^{*}(x, x)= & a_{n-1}\left(1-\tau_{1}\right)^{-2} \cdots\left(1-\tau_{s-1}\right)^{-2} \\
& \cdot\left(q_{n, r}^{\prime}(x) q_{n-1, r-2}(x)-q_{n, r}(x) q_{n-1, r-2}^{\prime}(x)\right) .
\end{aligned}
$$

Both these corollaries follow from the fact that $P_{k}$ are actually orthonormal polynomials and from the well-known properties of the zeros and the ChristoffelDarboux formula (2.6) shared by all orthogonal polynomials. To emphasize their importance in the study of quasi-orthogonal polynomials, we stated them in terms of $q_{n, k}$. Their applications to quadrature formulae are presented in the next section. For $r=3$ these corollaries are proved in [12], where Corollary 3.4 is proved using a different method.

\section{Positive QUAdRatURe Formula}

Using the symmetric matrix representation of quasi-orthogonal polynomials, we give our characterization of positive quadrature formulae in this section. For completeness, we give a complete proof for the following theorem, although part of the first half is standard.

Theorem 4.1. Let $q_{n, r}$ generate a $(2 n-r-1, n)$ quadrature formula $\mathscr{I}_{n, r}$. Then $\mathscr{I}_{n, r}$ is a positive quadrature formula if and only if $q_{n, r}$ is a quasiorthogonal polynomial of degree $n$, and order $r$, that has a positive symmetric matrix representation.

Proof. Let $q_{n, r}$ be a quasi-orthogonal polynomial that has a positive symmetric matrix representation. By Corollary 3.3, $q_{n, r}$ has $n$ distinct zeros, which we denote by $x_{1, n}, \ldots, x_{n, n}$. For a given function $f$ on $\mathbb{R}$, let $L_{n}(f)$ be the 
$n$th Lagrange interpolating polynomial based on the zeros of $q_{n, r}$. Then it is easy to verify that

$$
L_{n}(f, x)=\sum_{k=1}^{n} f\left(x_{k, n}\right) l_{k, n}(x), \quad l_{k, n}(x)=\frac{K_{n}^{*}\left(x, x_{k, n}\right)}{K_{n}^{*}\left(x_{k, n}, x_{k, n}\right)},
$$

where $K_{n}^{*}$ is defined in (3.9). Indeed, from (3.9) and (3.10) we have $l_{k, n} \in \Pi_{n-1}$ and

$$
l_{k, n}\left(x_{j, n}\right)=\delta_{k, j}, \quad 1 \leq k, j \leq n,
$$

which implies $L_{n}\left(f, x_{k, n}\right)=f\left(x_{k, n}\right)$. The integration of $L_{n}(f)$ leads to a quadrature formula for $d \alpha$,

$$
\mathscr{I}_{n, r}(f)=\int_{\mathbb{R}} L_{n}(f, x) d \alpha=\sum_{k=1}^{n} f\left(x_{k, n}\right)\left[K_{n}^{*}\left(x_{k, n}, x_{k, n}\right)\right]^{-1} .
$$

If $P \in \Pi_{2 n-r-1}$, then $P$ can be written uniquely as $P=g q_{n, r}+R$, where $g \in \Pi_{n-r-1}$ and $R \in \Pi_{n-1}$. Therefore, we have

$$
\mathscr{I}_{n, r}(P)=\sum_{k=1}^{n} R\left(x_{k, n}\right)\left[K_{n}^{*}\left(x_{k, n} x_{k, n}\right)\right]^{-1}=\mathscr{I}_{n, r}(R)=\int_{\mathbb{R}} R(x) d \alpha .
$$

Since $q_{n, r}$ is a quasi-orthogonal polynomial which is orthogonal to $\Pi_{n-r-1}$, we have that $\int g q_{n, r} d \alpha=0$. Thus,

$$
\mathscr{I}_{n, r}(P)=\int_{\mathbb{R}}\left[g(x) q_{n, r}(x)+R(x)\right] d \alpha=\int_{\mathbb{R}} P(x) d \alpha, \quad P \in \Pi_{2 n-r-1},
$$

which shows that $\mathscr{J}_{n, r}$ is a $(2 n-r-1, n)$ quadrature formula. Moreover, from (3.9) we clearly have that the quadrature weights $\lambda_{k, n}=\left[K_{n}^{*}\left(x_{k, n}, x_{k, n}\right)\right]^{-1}>$ $0,1 \leq k \leq n$. Thus, the quadrature formula $\mathscr{I}_{n, r}$ is a positive one.

On the other hand, suppose a $(2 n-r-1, n)$ quadrature (1.1) of $d \alpha$ exists. We let $q(x)=\left(x-x_{1, n}\right) \cdots\left(x-x_{n, n}\right)$. Then $q \in \Pi_{n}$ and for every polynomial $p \in \Pi_{n-r-1}$ we have

$$
\int_{\mathbb{R}} q(x) p(x) d \alpha=\sum_{k=1}^{n} q\left(x_{k, n}\right) p\left(x_{k, n}\right) \lambda_{k, n}=0 ;
$$

therefore, $q$ is orthogonal to $\Pi_{n-r-1}$ with respect to $d \alpha$, and it is a quasiorthogonal polynomial of the form (1.3). Each $f \in \Pi$ has a unique representation $f=g q+R_{q}(f)$, where $g \in \Pi, R_{q}(f) \in \Pi_{n-1}$. Following [9], we define a linear functional $\mathscr{L}_{q}: \Pi \mapsto \mathbb{R}$ associated with $q$ as follows:

$$
\mathscr{L}_{q}: \Pi \mapsto \mathbb{R}, \quad \mathscr{L}_{q}(f)=\int_{\mathbb{R}} R_{q}(f, x) d \alpha .
$$

Since $q$ generates a $(2 n-r-1, n)$ quadrature, this linear functional is positive on $\Pi_{2 n-1}$. This fact is proved in [9, p. 397]; for completeness we reproduce the proof here. Every nonnegative polynomial $f \in \Pi_{2 n-1}$ can be written as $f=p_{1}^{2}+p_{2}^{2}$ for some $p_{1}, p_{2} \in \Pi_{n-1}$. Such an $f$, if not identical zero, cannot vanish at all $n$ nodes of the quadrature formula. Hence, by $R_{q}\left(f, x_{k, n}\right)=$ $f\left(x_{k, n}\right)$ we obtain

$$
\mathscr{L}_{q}(f)=\int_{\mathbb{R}} R_{q}(f, x) d \alpha=\int_{\mathbb{R}}(f-g q)(x) d \alpha=\sum_{k=1}^{n} \lambda_{k n} f\left(x_{k, n}\right)>0,
$$


and $\mathscr{L}_{q}$ is positive. Moreover, for every $p \in \Pi_{n-1}$ we have $p q \in \Pi_{2 n-1}$, and $\mathscr{L}_{q}(p q)=0$. This means that $q$ is orthogonal to $\Pi_{n-1}$ with respect to the linear functional $\mathscr{L}_{q}$. By the Gram-Schmidt orthonormalization process, the positivity of $\mathscr{L}_{q}$ on $\Pi_{2 n-1}$ implies the existence of polynomials $q_{0}, \ldots, q_{n-1}$ that are orthonormal with respect to $\mathscr{L}_{q}$. Moreover, $q_{k}=\gamma(q) x^{k}+\cdots$, where $\gamma_{k}(q)>0$. Since $\mathscr{L}_{q}(f)=\int f d \alpha$ for $f \in \Pi_{2 n-s}$, we also have $q_{k}=p_{k}(d \alpha)$, $0 \leq k \leq n-[r / 2]$. These orthonormal polynomials satisfy a three-term relation, which can be written as

$$
q_{k+1}=\frac{1}{a_{k}^{\prime}}\left(x-b_{k}^{\prime}\right) q_{k}-\frac{1}{a_{k-1}^{\prime}} q_{k-1}, \quad 1 \leq k \leq n-2,
$$

where by the orthogonality and the positivity of $\mathscr{L}_{q}$ we have

$$
a_{k}^{\prime}=\mathscr{L}_{q}\left(x q_{k} q_{k+1}\right)=\frac{\gamma_{k}(q)}{\gamma_{k+1}(q)} \mathscr{L}_{q}\left(q_{k+1}^{2}\right)=\frac{\gamma_{k}(q)}{\gamma_{k+1}(q)}>0, \quad 0 \leq k \leq n-2 .
$$

The polynomial $q \in \Pi_{n}$ can be written as $q=\gamma_{n-1}^{-1} x q_{n-1}+\sum_{k=0}^{n-1} c_{k} q_{k}$. By the orthogonality of $q_{k}, 0 \leq k \leq n-1$, and $q$ with respect to $\mathscr{L}_{q}$, we obtain that

$$
q=\gamma_{n-1}^{-1}\left(x q_{n-1}-b_{n-1}^{\prime} q_{n-1}-a_{n-2}^{\prime} q_{n-2}\right),
$$

where $b_{n-1}^{\prime}=\mathscr{L}_{q}\left(x q_{n-1}^{2}\right)$. From this equality and (4.2) it is readily seen that $q$ admits a symmetric matrix representation as in (3.2) with $a_{k}^{*}=a_{k}^{\prime}$. Since all $a_{k}^{\prime}>0,0 \leq k \leq n-2$, the representation is positive. Let $\tau_{k}=1-a_{k}^{\prime} / a_{k}$. Then $a_{k}^{\prime}=a_{k}\left(1-\tau_{k}\right)$. We can also define $\sigma_{k}, 1 \leq k \leq s$, by $b_{n-s+k-1}^{\prime}=$ $b_{n-s+k-1}-a_{n-s+k-1} \sigma_{k}$. Thus, the matrix representation takes exactly the form of (3.2).

Using this theorem, we can state several corollaries. The first comes directly from the proof of the theorem; it provides an explicit formula for the weights of a positive quadrature formula.

Corollary 4.2. If $\mathscr{I}_{n, r}$ in (1.1) is a positive quadrature formula generated by $q_{n, r}$, then its weights are given by

$$
\begin{aligned}
\lambda_{k, n} & =\left[K_{n}^{*}\left(x_{k, n}, x_{k, n}\right)\right]^{-1} \\
& =a_{n-1}^{-1}\left(1-\tau_{1}\right)^{2} \cdots\left(1-\tau_{s-1}\right)^{2}\left[q_{n, r}^{\prime}\left(x_{k, n}\right) q_{n-1, r-2}\left(x_{k, n}\right)\right]^{-1} .
\end{aligned}
$$

We note that if one of $a_{k}^{*}=0$, then $\tau_{k}=1$, and the right-hand side of the equation (4.3) becomes zero. Thus, $\left[K_{n}^{*}\left(x_{k, n}, x_{k, n}\right)\right]^{-1}=0$, and they cannot be weights for any quadrature formula. Actually, in this case, $q_{n, r}$ is not a quasi-orthogonal polynomial; it will not generate a $(2 n-r-1, n)$ formula, positive or not.

The next corollary of Theorem 4.1 is of particular interest, for it provides another characterization of positive $(2 n-r-1, n)$ quadrature formula, which states that such a formula is actually a Gaussian quadrature for some nonnegative measure.

Corollary 4.3. Let $n \in \mathbb{N}$ be fixed. If $\mathscr{I}_{n, r}(d \alpha)$ in (1.1) is a $(2 n-r-1, n)$ positive quadrature formula for $\alpha \in \mathscr{M}$, then it is a Gaussian quadrature formula for another measure $\mu_{n} \in \mathscr{M}$.

Proof. If $\mathscr{I}_{n, r}(d \alpha)$ is a $(2 n-r-1, n)$ positive quadrature formula, then by Theorem 4.1 it is generated by a quasi-orthogonal polynomial $q_{n, r}$ of degree 
$n$ which has a positive symmetric matrix representation. Because of such a representation, by Theorem 3.2, $q_{n, r}$ is a member of a family of orthogonal polynomials with respect to a nonnegative measure $\mu_{n}$. By (2.7), the $n$th Gaussian quadrature formula with respect to $\mu_{n}$ is

$$
\mathscr{F}_{n}\left(d \mu_{n}, f\right)=\sum_{k=1}^{n} f\left(x_{k, n}\right)\left[K_{n}^{*}\left(x_{k, n}, x_{k, n}\right)\right]^{-1},
$$

where $x_{k, n}$ are zeros of $q_{n, r}$. By Corollary 4.2 , we see that this is exactly the quadrature $\mathscr{I}_{n, r}(d \alpha)$.

In our definition of a $(2 n-r-1, n)$ formula, we have $0 \leq r \leq n$. Since each positive quadrature is at least $(n-1, n)$ type if its nodes are distinct, the union of the class of $(2 n-r-1, n)$ positive quadrature formulae for $0 \leq r \leq n-1$ is the class of all positive quadrature formulae. Therefore, we have the following characterization of positive quadrature formulae, which we state as a theorem.

Theorem 4.4. Let $\alpha \in \mathscr{M}$ and $n \in \mathbb{N}$ be fixed. A quadrature formula $\mathscr{I}_{n, r}(d \alpha)$ with distinct nodes is positive if, and only if, it is a Gaussian quadrature formula for some measure $\mu_{n} \in \mathscr{M}$. Moreover, if $x_{1, n}, \ldots, x_{n, n}$ are the nodes of the quadrature, then the polynomial $q_{n}(x)=\left(x-x_{1, n}\right) \cdots\left(x-x_{n, n}\right)$ has a positive symmetric matrix representation.

For example, the $(2 n-2, n)$ quadrature formula generated by $q_{n}(u)$ in (3.5), for the Chebyshev weight function of the second kind $u$, is also the Gaussian quadrature formula for the weight function $u^{*}$ in (3.6). The $(2 n-$ $3, n)$ quadrature formula generated by $q_{n}(v)$ in (3.7), for the Chebyshev weight function of the first kind $v$, is also the Gaussian quadrature formula for the weight function $v^{*}$ in (3.8).

However, we remark that these examples are somewhat special, as the measures $u^{*}$ and $v^{*}$ are independent of $n$. In general, the measure $\mu_{n}$ in Theorem 4.4 has to depend on $n$. To show this, we recall the definition of the Chebyshev quadrature formula. A quadrature formula is called a Chebyshev quadrature if it is equally weighted,

$$
\int_{\mathbb{R}} f(x) d \alpha=A_{n} \sum_{k=1}^{n} f\left(x_{k, n}\right), \quad f \in \Pi_{n-1} .
$$

If a weight function $w$ admits a Chebyshev quadrature formula for every positive integer $n$, we say that the weight function $w$ has property $\mathrm{T}$. It is well known that the Chebyshev weight function $v(x)=\left(1-x^{2}\right)^{-1 / 2}$ has property $\mathrm{T}$, and the corresponding quadrature is the Gaussian quadrature. Moreover, it is the only weight function all of whose Gaussian quadratures is equally weighted (cf. [4]). There are other weight functions that have property $\mathrm{T}$ (cf. $[4,11]$ and the references therein). As an example, we mention $w_{0}(x)=\left(1-x^{2}\right)^{-1}(1+b x)$, $|b|<1 / 4$ [11]. Clearly, the Chebyshev quadrature formulae are positive ones. If all measures $\mu$ in Theorem 4.4. were independent of $n$, then $\mu^{\prime}$ corresponding to $w_{0}$ would be a weight function whose Gaussian quadrature formulae are equally weighted for all $n$, which, however, would contradict the uniqueness of the Chebyshev weight function, for it is the only one having such a property. 


\section{CONSTRUCTION OF POSITIVE QUADRATURE FORMULAE}

Theorem 4.1 characterizes all positive $(2 n-r-1, n)$ formulae. Moreover, our discussion in the previous two sections, notably Corollary 4.2 and Theorem 3.1, actually provides a very efficient method to construct such a formula. Indeed, by Theorem 3.1 and Corollary 3.3, the zeros of $q_{n, r}$ are distinct eigenvalues of the symmetric matrix $T_{n, r}$. Thus, for a given $T_{n, r}$, they can be found numerically using one of the existing methods of computing the eigenvalues, for example, the $Q R$ method. Once the zeros are found, the quadrature weights can be computed by the use of (4.3) in Corollary 4.2. We summarize this construction process in the following.

Theorem 5.1. Let $q_{n, r}$ be a quasi-orthogonal polynomial that has a positive symmetric matrix representation (3.2). Let $\mathscr{I}_{n, r}$ be the positive $(2 n-r-1, n)$ quadrature formula generated by $q_{n, r}$. Then the nodes of the quadrature formula are the eigenvalues of the matrix $T_{n, r}$, and the weights of the quadrature formula are given by formula (4.3).

From this point of view, in the study of positive quadrature formulae, it is very natural to define the quasi-orthogonal polynomial $q_{n, r}$ by means of the matrix (3.1). However, in the literature and perhaps in some applications, the $q_{n, r}$ is usually given in the form of

$$
q_{n, r}=p_{n}(d \alpha)+\rho_{1} p_{n-1}(d \alpha)+\cdots+\rho_{r} p_{n-r}(d \alpha) .
$$

As we pointed out in $\S 3$, not every $q_{n, r}$ given in this form has a symmetric matrix representation. But our Theorem 4.1 asserts that if $q_{n, r}$ generates a quadrature formula, then it must have a positive symmetric matrix representation. In [12], we discussed the symmetric matrix representation of $q_{n, r}$ and used such representations to study the quadrature formula. But our discussion there takes (5.1) as the starting point, and we found, for $r=2,3$, the class of quasi-orthogonal polynomials in (5.1) that have a symmetric matrix representation. This requires explicit formulae for $\tau_{k}$ and $\sigma_{k}$ in terms of $\rho_{k}$. The complexity of these formulae for $r>3$ is the main reason why we restricted our discussion in [12] to $r=3$. The formulae developed in [12] allow us to state the characterization in Theorem 4.1 in terms of $\rho_{k}$. We give the results in the following.

For $r=1$, the quasi-orthogonal polynomial is $q_{n, 1}=p_{n}(d \alpha)+\rho_{1} p_{n-1}(d \alpha)$, and it generates a $(2 n-2, n)$ quadrature formula with respect to $d \alpha$. In this case, it is well known that all $(2 n-2, n)$ quadrature formulae are positive, and $q_{n, 1}$ has a positive symmetric matrix representation with $\sigma_{1}=\rho_{1}$.

We list the next two cases as corollaries. The case $r=3$ actually includes the case $r=2$, since $q_{n, 3}$ becomes $q_{n, 2}$ when $\rho_{3}=0$ and our definition of the matrix representation is consistent with this fact. However, we still state these two cases separately in the following, as we feel that our results may be better illustrated this way. The derivation of the formulae below are carried out in [12].

Corollary 5.2. Let $q_{n, 2}$ be a quasi-orthogonal polynomial defined by

$$
q_{n, 2}=p_{n}(d \alpha)+\rho_{1} p_{n-1}(d \alpha)+\rho_{2} p_{n-2}(d \alpha) .
$$

Then the $(2 n-3, n)$ quadrature formula generated by $q_{n, 2}$ is positive if, and 
only if, $\rho_{2} \leq a_{n-2} / a_{n-1}$. Moreover, $q_{n, 2}$ has a positive symmetric representation with

$$
\sigma_{2}=\rho_{1}, \quad \tau_{1}=1-\sqrt{1-\frac{a_{n-1} \rho_{2}}{a_{n-2}}} .
$$

Corollary 5.3. Let $q_{n, 3}$ be a quasi-orthogonal polynomial defined by

$$
q_{n, 3}=p_{n}(d \alpha)+\rho_{1} p_{n-1}(d \alpha)+\rho_{2} p_{n-2}(d \alpha)+\rho_{3} p_{n-3}(d \alpha) \text {. }
$$

Then the $(2 n-4, n)$ quadrature formula generated by $q_{n, 3}$ is positive if, and only if,

$$
A_{n}:=1-\frac{a_{n-1}}{a_{n-2}} \rho_{2}+\frac{a_{n-1} \rho_{3}}{a_{n-2} a_{n-3}}\left(b_{n-2}-b_{n-1}+a_{n-1} \rho_{1}\right)-\left(\frac{a_{n-1}}{a_{n-3}}\right)^{2} \rho_{3}^{2}>0 .
$$

Moreover, $q_{n, 3}$ has a positive symmetric representation with

$$
\sigma_{1}=\frac{a_{n-1}}{a_{n-3}} \rho_{3}, \quad \sigma_{2}=\rho_{1}-\frac{a_{n-2}}{a_{n-3}} \rho_{3}, \quad \tau_{1}=1-\sqrt{A_{n}\left(\rho_{1}, \rho_{2}, \rho_{3}\right)} .
$$

The complexity of the characterization of the positive quadrature formulae in terms of $\rho_{k}$ in (5.1) increases rapidly as $r$ increases. Therefore, for $r>3$, our characterization of a positive quadrature for $q_{n, r}$ given in the form of (5.1) becomes difficult to apply.

Often in the applications, we want to have some knowledge about the location of the quadrature nodes. Suppose that the measure $\alpha$ has compact support $[a, b]$; we would like to know when a positive $(2 n-r-1, n)$ quadrature formula with respect to $d \alpha$ has all its nodes inside $[a, b]$. By Theorem 4.1, this is equivalent to asking when the zeros of the quasi-orthogonal polynomial $q_{n, r}$ are located inside $[a, b]$. This question has been addressed in [10, p. 46] for $r=1$, in [6] for $r=2$, in [12] for $r=3$, and in [7, 8, 11] for general $r$. We shall follow the approach used in [12], which is based on a representation of the largest (and the smallest) eigenvalue of a symmetric tridiagonal matrix in [2], (see also [5]). The result that we need is as follows.

Theorem 5.4. Let $J_{n}$ be any truncated Jacobi matrix of the form (2.3). Let the largest and the smallest eigenvalue of $J_{n}$ be denoted by $\xi_{n}$ and $\xi_{1}$, respectively. Then with $\chi=\left\{\chi_{1}, \ldots, \chi_{n+1}\right\}$ ranging over all sequences such that $\chi_{1}=\infty$, $\chi_{n+1}=0$, and $\chi_{k}>0,2 \leq k \leq n$, one has

$$
\xi_{1}=\max _{\chi} \min _{1 \leq k \leq n}\left\{b_{k-1}-\frac{a_{k-2}^{2}}{\chi_{k}}-\chi_{k+1}\right\}
$$

and

$$
\xi_{n}=\min _{\chi} \max _{1 \leq k \leq n}\left\{b_{k-1}+\frac{a_{k-2}^{2}}{\chi_{k}}+\chi_{k+1}\right\} .
$$

In [2], this theorem was stated in terms of the entries in a nonsymmetric tridiagonal matrix corresponding to the monic orthogonal polynomials. For convenience we restated it here in terms of the symmetric matrix which corresponds to the orthonormal polynomials. Let the zeros of $q_{n, r}$ be denoted by $t_{k, n}$ and ordered by $t_{1 n}<t_{2 n}<\cdots<t_{n n}$. If $q_{n, r}$ generates a positive quadrature formula, then it has a positive symmetric matrix representation. We apply Theorem 5.4 to this matrix representation of $q_{n, r}$. 
Theorem 5.5. Suppose that $\alpha$ is supported on $[a, b]$ and $q_{n, r}$ has a positive symmetric matrix representation. Then the zeros of $q_{n, r}$ are all located inside $[a, b]$ if and only if

$$
q_{n-k, r-2 k}(b)>0, \quad(-1)^{n-k} q_{n-k, r-2 k}(a)>0, \quad 0 \leq k \leq\left[\frac{r}{2}\right],
$$

where the polynomials $q_{n-k, r-2 k}$ are related to $q_{n, r}$ as defined in (3.3).

Proof. To simplify the notation, we use the notation $Q_{k}$ in (3.4). The condition (5.4) in terms of this notation becomes

$$
Q_{n-k}(b)>0, \quad(-1)^{n-k} Q_{n-k}(a)>0, \quad 0 \leq k \leq[r / 2] .
$$

Since $Q_{n-[r / 2]-1}=p_{n-[r / 2]-1}$, by (2.5) we obtain that (5.4) is equivalent to

$$
\frac{Q_{k}(b)}{Q_{k-1}(b)}>0, \quad \frac{Q_{k}(a)}{Q_{k-1}(a)}<0, \quad n-\left[\frac{r}{2}\right] \leq k \leq n .
$$

Suppose now that conditions (5.5) are satisfied. By Theorem 4.1, $t_{k, n}$ are eigenvalues of a symmetric tridiagonal matrix in (3.1). We apply Theorem 5.4 to the polynomial $q_{n, r}$ by choosing

$$
\chi_{k}=-a_{k-2}^{*} \frac{Q_{k-1}(a)}{Q_{k-2}(a)}>0, \quad 1 \leq k \leq n,
$$

where the positivity of $\chi_{k}$ follows from (5.5) and from $a_{k-2}^{*}>0$. With this choice we get rid of the maximum in (5.2). Let the expression inside the braces be defined by $t_{k}^{*}$. Using the three-term relation, we have for $1 \leq k \leq n-1$,

$$
\begin{aligned}
t_{k}^{*} & =b_{k-1}^{*}-\frac{\left(a_{k-2}^{*}\right)^{2}}{\chi_{k}}-\chi_{k+1} \\
& =b_{k-1}^{*}+a_{k-2}^{*} \frac{Q_{k-2}(a)}{Q_{k-1}(a)}+a_{k-1}^{*} \frac{Q_{k}(a)}{Q_{k-1}(a)}=a .
\end{aligned}
$$

Since by definition of $Q_{n}$ we have

$$
a_{n-1} Q_{n}=\left(x-b_{n}^{*}\right) Q_{n-1}-a_{n-2}^{*} Q_{n-2},
$$

the $n$th inequality becomes

$$
t_{n}^{*}=b_{n-1}^{*}+a_{n-2}^{*} \frac{Q_{n-2}(a)}{Q_{n-1}(a)}=a-a_{n-1} \frac{Q_{n}(a)}{Q_{n-1}(a)}>a .
$$

Therefore, from (5.2), we conclude $t_{1, n} \geq \min _{k} t_{k}^{*} \geq a$. The case of the largest zero $t_{n, n} \leq b$ is proved similarly.

On the other hand, suppose all zeros of $Q_{n}=q_{n, r}$ are inside $(a, b)$. By Theorem 3.2, the zeros of $Q_{k}$ and $Q_{k-1}$ separate each other. Therefore, all zeros of $Q_{k}, 0 \leq k \leq n-1$, are inside $(a, b)$. By (3.3), $Q_{k}(x)=\gamma_{k} x^{k}$ $+\cdots$ and $\gamma_{k}>0$, so that $\operatorname{sign} Q_{k}(b)=\operatorname{sign} Q_{k}(\infty)=1$. Therefore, the first inequality of $(5.4)$ holds. Similarly, we have sign $Q_{k}(a)=(-1)^{k}$, which implies the second inequality of (5.4).

This theorem was proved in [9] using Sturm's Theorem. For $q_{n, 1}$ the condition (5.4) is given in [12, p. 46]; for $q_{n, 2}$, these conditions are equivalent to those given in [6]. See also $[7,8]$ for a different characterization. Our method follows what we used for the case $r=3$ in [12], which is different from the previous ones. 


\section{BIBLIOGRAPHY}

1. T. S. Chihara, An introduction to orthogonal polynomials, Math. Appl., vol. 13, Gordon \& Breach, New York, 1978.

2. E. A. Van Doorn, Representations and bounds for zeros of orthogonal polynomials and eigenvalues of sign-symmetric tri-diagonal matrices, J. Approx. Theory 51 (1987), 254-266.

3. G. Freud, Orthogonal polynomials, Pergamon Press, Oxford, 1971.

4. W. Gautschi, Advances in Chebyshev quadrature, Lecture Notes in Math., vol. 506, SpringerVerlag, New York and Berlin, 1976, pp. 100-121.

5. J. Gilewicz and E. Leopold, On the sharpness of results in the theory of location of zeros of polynomials defined by three term recurrence relations, Polynômes Orthogonaux et Applications, Lecture Notes in Math., vol. 1171, Springer-Verlag, New York and Berlin, 1985, pp. 259-266.

6. C. A. Micchelli and T. J. Rivlin, Numerical integration rules near Gaussian formulas, Israel J. Math. 16 (1973), 287-299.

7. F. Peherstorfer, Characterization of positive quadrature formulas, SIAM J. Math. Anal. 12 (1981), 935-942.

8. $\ldots$, Characterization of quadrature formulas. II, SIAM J. Math. Anal. 15 (1984), 10211030.

9. H. J. Schmid, A note on positive quadrature rules, Rocky Mountain J. Math. 19 (1989), 395-404.

10. G. Szegö, Orthogonal polynomials, 4th ed., Amer. Math. Soc. Colloq. Publ., vol. 23, Amer. Math. Soc., Providence, RI, 1975.

11. Y. Xu, Weight functions for Chebyshev quadrature, Math. Comp. 53 (1989), 297-302.

12. _ Quasi-orthogonal polynomials, quadrature, and interpolation, J. Math. Anal. Appl. (to appear).

Department of Mathematics, University of Oregon, Eugene, Oregon 97403-1222

E-mail address: yuan@bright.uoregon.edu 